\title{
"Es muss net alles immer nur auf Gewinn auf'baut sein." Zusammenfassung und Ausblick
}

Elisabeth Kosnik, Andrea Heistinger, Gabriele Sorgo

Wie aktuelle Studien belegen, liegt Österreich sowohl bei der Produktion als auch beim Konsum ökologischer Produkte im EU-Spitzenfeld. ${ }^{1}$ Jede fünfte Landwirtschaft in Österreich wird mittlerweile ökologisch bewirtschaftet. Gleichzeitig dominieren wenige Supermarktketten den Vertrieb ökologischer Waren in Österreich. ${ }^{2}$ Ökologische Betriebe unterliegen längst dem Wachstums- und Rationalisierungstrend, was zunehmend zu einer Kritik an dieser »Konventionalisierung « führt, wie in den Kapitel von Andrea Heistinger und Elisabeth Kosnik nachgezeichnet wird. Diese Entwicklung hin zu einer konventionalisierten eco-entrepreneurial agriculture - in Anlehnung an den Begriff der entrepreneurial agriculture des Agrarsoziologen Jan Douwe van der Ploeg - ist allerdings kein flächendeckender Trend. ${ }^{3}$ Auch, oder gerade, im ökologischen Bereich gibt es Bäuerinnen und Bauern, die dem Trend des»Wachsen oder Weichen« kritisch gegenüberstehen und nach alternativen Wegen der Produktion und Distribution suchen. Diese Landwirt*innen standen im Fokus der vorliegenden Studie. Dabei sollte Öko-Landwirtschaft in ihren ökonomischen, sozialen, lokalen und ökologischen Dimensionen betrachtet werden, wobei auch nicht-menschliche Akteur*innen - im

1 Vgl. European Commission 2019.

2 Vgl. Bio Austria Statistik.

3 Vgl. Ploeg 2018b. 
Sinne von foodwebs, wie Gabriele Sorgo in ihrem Kapitel darstellt - miteinbezogen werden. Dabei zeigt sich, dass es gerade die affektiven Beziehungen zu Bodenlebewesen, Tieren und Menschen sind, die mit einer produktivistischen Denkweise unvereinbar sind. Die seit der Covid19 Pandemie rasant gestiegene Nachfrage nach regionalen Lebensmitteln, sowie der steigende Anteil an Direktvermarktung, scheinen diesen Produzent*innen Recht zu geben. ${ }^{4}$

Die Ergebnisse des vorliegenden Bandes basieren auf einem zwischen 2017-2019 durchgeführten transdisziplinären Forschungsprojekt. Im Mittelpunkt stand dabei die Frage, wer die Landwirt*innen sind, die neue Wege der Produktion und Distribution im ökologischen Sektor gehen. Gleichzeitig sollte dabei die Frage betrachtet werden, wie eine sozial und ökologisch nachhaltige und zugleich lebenswerte Landwirtschaft für die Bewirtschafter*innen aussehen könnte. Erforscht wurden dazu zertifiziert ökologische Vollerwerbsbetriebe, die Grundnahrungsmittel für Menschen produzieren und diese, zumindest teilweise, abseits von Großkunden regional vertreiben. Die qualitativen Erhebungen wurden auf vier ökologischen Landwirtschaftsbetrieben in strukturschwachen, ländlich geprägten Regionen im Osten Österreichs durchgeführt.

Methodisch basiert die Forschung auf dem sinnverstehenden Ansatz der Soziologie, der davon ausgeht, dass Menschen einerseits handlungsfähige Akteur*innen sind, ihr Handlungsspielraum aber andererseits durch orts- und zeitspezifische Bedingungen geprägt ist. Die Herangehensweise stützt sich auf die Einarbeitung historischer und familiensoziologischer Daten. Die Datenerhebung erfolgte durch qualitative Interviews mit den Betriebsleiter*innen, ergänzt durch mehrfache Besuche auf den vier untersuchten Höfen. Insbesondere wurden die Familiengeschichten der Landwirt*innen erhoben, basierend auf der familiensoziologisch-historischen Methode der Genogrammanalyse nach Bruno Hildenbrand. ${ }^{5}$ Im Rahmen des Projektes wurden daher nicht nur

4 Vgl. European Commission 2020; Bio Austria Statistik; Bio Austria Presseaussendung (veröffentlicht am 07.09.2020).

5 Vgl. Hildenbrand 2018. 
die Betriebsleiter*innen interviewt, sondern darüber hinaus ihre (insgesamt neun) Familiengeschichten bis zur Großelterngeneration - daher bis an den Anfang des 20. Jahrhunderts - rekonstruiert.

Die Betriebe (theoretical sampling) wurden gemäß einer im Verlauf des Forschungsprozesses festgelegten Kontrastierung ausgewählt. Dass es sich bei allen Fallbeispielen um Betriebsleiterpaare handelt, die zusammen mit ihren Kindern am Betrieb leben, war keine von uns gewählte Voraussetzung, sondern stellt eines der Ergebnisse der vorliegenden Forschung dar. Dies ist in Anbetracht der fortschreitenden Abwanderung junger Menschen und insbesondere von jungen Frauen aus ländlichen Gebieten in ganz Europa bemerkenswert. ${ }^{6}$ Ziel der Methode im Kontext unseres Forschungsprojekts war es, Dispositionen gegenüber Herausforderungen innerhalb von Familien zu rekonstruieren und davon erfolgreiche Strategien für Arbeits- und Alltagsleben ökolandwirtschaftlicher Betriebsleiter*innen abzuleiten. Auf diese Weise können historische, sozio-ökonomische, ökologische und regionale $\mathrm{Be}$ dingungen zu den individuellen Vorgangsweisen in Beziehung gesetzt werden. Gleichzeitig wird dadurch aufgezeigt, dass es keinen homogenen Öko-Sektor gibt. Die Akteur*innen, ihre Handlungsstrategien und Motivationen, sind vielfältig.

Als Forschungsgebiet diente primär das ländlich geprägte Flachund Hügelland der als Oststeiermark bezeichneten Region. Dieses Gebiet hat durch seine geographische Lage als historisches Grenzgebiet im Osten eine Sonderentwicklung erfahren. Die Industrialisierung erreichte die Region, verglichen mit anderen europäischen Gebieten, erst spät, wurde dann aber umso energischer vorangetrieben. War die Oststeiermark aufgrund der abgeschiedenen Lage und der fehlenden Verkehrserschließung weit bis ins 20. Jahrhundert auf Selbstversorgung und einen regionalen Wirtschaftskreislauf angewiesen, setzte ab der Mitte des 20. Jahrhunderts eine Entwicklung ein, die Karl Polanyi als Entbettung der wirtschaftlichen Verhältnisse bezeichnet. ${ }^{7}$ Diese Entwicklung wurde von den Großeltern-, bzw. Elterngenerationen der er-

6 Vgl. Weber und Fischer 2012; Leibert und Wiest 2016.

7 Vgl. Polanyi 1977. 
erbten Höfe unter den Fallbeispielen mitgetragen. Auf den Landwirtschaften wurde Produktion und Distribution rationalisiert, die kleinen und vielfältigen Betriebe verschwanden zusehends und die Bevölkerung wanderte in urbane Regionen $\mathrm{ab}$ - ein Trend, der sich bis heute fortsetzt.

Heute hat die Region vollen Verkehrsanschluss und ist gänzlich mobilisiert, aber auch Kommunikationstechnologien, insbesondere das Internet, bieten den Höfen neue Möglichkeiten. Wie die Forschungsergebnisse aufzeigen, stehen die Betriebsleiter*innen trotz dieser Voraussetzungen den globalen Lebensmittelsystemen - von der Primärerzeugung, über multinationale Betriebsmittelkonzerne und global agierende Lebensmittelkonzerne, hin zu Lebensmittelspekulationen an den Agrarbörsen usw. - kritisch bis ablehnend gegenüber. Manche ökologische Landwirt*innen der Pioniergeneration in Österreich - wie Familie Danninger, die seit den 1990er Jahren Supermärkte mit ihren Produkten beliefern - betrachten die Eingliederung der ökologischen Produktion in diese Lebensmittelsysteme heute kritisch. Die Landwirt"innen unserer Forschung investieren hingegen bewusst in langfristige Beziehungen abseits dieser Systeme - in Menschen sowie in andere Mitlebewesen wie Nutztiere, Pflanzen und Bodenlebewesen. Sie setzen mit ihren Alltagspraxen Ansätze um, die von Kritiker*innen einer konventionellen Landwirtschaft seit vielen Jahrzehnten eingefordert werden. ${ }^{8}$ Ihre gemeinwohlorientierten, vernetzten, holistischen Handlungspraktiken und Denkweisen umschreiben die Autorinnen in diesem Band mit dem Begriff der sorgsamen Landwirtschaft (caring agriculture).

In einer Mitteilung an das Europäische Parlament vom Mai 2020 stellt die Europäische Kommission fest, dass der »Ruf nach kürzeren Lieferketten während des Covid-19-Ausbruchs lauter geworden « ist. ${ }^{9}$ Die Mitteilung unterstreicht die Bedeutung der ökologischen Landwirtschaft für ein nachhaltiges Lebensmittelsystem und von kurzen Lieferketten, welche die Resilienz regionaler Lebensmittelsysteme fördern.

8 Vgl. Meadows u.a. und Club of Rome 1972.

9 European Commission 2020, S. 3. 
Die Landwirt*innen unserer Studie haben diesen Trend übernommen und setzen auf Direktvermarktung - wie Bauernmärkte, ab Hof Verkauf und CSAs - oder beliefern Foodcoops sowie regionale Gastgewerbe und Läden. Familie Bergmann etwa hat sich ganz bewusst dazu entschieden, ausschließlich nach lokalen Mitgliedern für ihre CSA zu suchen. Durch den persönlichen Kontakt konnten sich die Direktvermarkter*innen einen stabilen Kundenstock schaffen.

Die jüngere Generation von Landwirt*innen versucht sich bewusst vom Mainstream abzuheben und autonom handlungsfähig zu bleiben. ${ }^{10}$ Generell jedoch betonen alle Landwirt*innen in unserer Studie ihr Bedürfnis nach einem selbstbestimmten Leben, weshalb fast alle unter ihnen Kredite genauso zu vermeiden versuchen, wie Verflechtungen mit vor- und nachgelagerten Industrien, was sie im Sinne Ploegs als peasants auszeichnet. ${ }^{11}$ Gleichzeitig spielen Selbstverwirklichung, Kreativität, die Möglichkeit, ihre Fähigkeiten sinnvoll einzubringen und die Freude an der Arbeit mit der Natur eine wichtige Rolle. Damit beschreiben Betriebsführer*innen ihre Vorstellungen von einem guten Leben, von sinnvoller Arbeit und von hochwertigen Lebensmitteln. So erzählt Herr Bergmann (der auf einer konventionellen Landwirtschaft aufgewachsen ist und in einer Landwirtschaftsschule ausgebildet wurde) von der genussvollen Erfahrung, zum ersten Mal in seinem Leben mit den eigenen Händen direkt in der Erde zu arbeiten, nachdem der Hof auf ökologische Gemüsewirtschaft umgestellt wurde (Interview am 2.3.2018).

Ausgehend von den Fallanalysen kann aufgezeigt werden, dass für die Betriebsleiter*innen eine ökologisch verträgliche Produktionsweise im Mittelpunkt steht, die Tierwohl, Bodenfruchtbarkeit, Biodiversität und Schadstoffminimierung fördert. Entgegen den Rationalisierungsbestrebungen seit dem 20. Jahrhundert weigern sich diese Akteur*innen der Öko-Landwirtschaft, ihre Höfe - den Boden wie ihre Nutztiere - als austauschbare Rohstoffe zu betrachten. Die Ziegenbäuerin Frau Conrad meint dazu: "Es muss net alles immer nur auf Gewinn auf'baut

10 Vgl. Milone und Ventura 2014; Kaser u.a. 2003.

11 Vgl. Ploeg 2018b. 
sein«(Interview am 5.7.2018). Die Methoden des Landbaus und der Tierhaltung gehen bei allen Interviewpartner*innen über die gesetzlichen Öko-Standards der Europäischen Union hinaus. Motiviert, die Gesundheit von Boden, Pflanzen, Tieren und Menschen zu fördern, betreiben die Befragten eine sorgsame Landwirtschaft.

Aus dieser Sicht wird erkennbar, dass die klassische wirtschaftstheoretische Unterscheidung von produktiver und reproduktiver Arbeit in Bezug auf eine sorgsame Landwirtschaft nicht haltbar ist. In einer sorgsamen Landwirtschaft wird der Mensch nicht nur als Abnehmer und Nutznießer der Leistungen von Boden und Bodenlebewesen verstanden, sondern als Teil einer Gemeinschaft, was Begriffe wie Puig de la Bellacasas human-soil communities in ähnlicher Weise beschreibt wie Ploegs co-production. ${ }^{12}$ Seit den Anfängen ökologischer Landbaumethoden wird von Praktiker*innen der Wunsch nach ökonomischer und sozialer Wertschätzung für diese Form der zeit- und arbeitsintensiven Produktionsweise geäußert, sowie eine faire Entlohnung für diese landwirtschaftliche care-Arbeit gefordert. ${ }^{13}$ Die Unsichtbarkeit der reproduktiven Sorgetätigkeit stellt ein grundsätzliches Problem unserer Gesellschaft dar, wie die Kapitel von Kosnik und Sorgo aufzeigen. Mit Hilfe qualitativer Forschungsmethoden lassen sich so geschaffene Mehrwerte jedoch erkennen. Denn Subsistenzarbeit heißt auch, langfristige Beziehungen herzustellen und Menschen und ihre Umwelten im Sinne der foodwebs - als Gemeinschaften zu verstehen.

Von den Behörden fühlen sich die Betriebsleiter*innen jedoch oft behindert, gerade, wenn es darum geht, gänzlich neue Methoden - etwa artgerechte Tierhaltung oder eine Tierwohl-orientierte, stressfreie Schlachtung - anzuwenden (Kapitel Heistinger, Kapitel Kosnik). Von staatlicher Seite wird diese care-Arbeit einer sorgsamen Landwirtschaft in Förderungen wie Flächenprämien für umweltschonend(er)e landwirtschaftliche Praktiken umgerechnet, als bürokratisch geregelte, standardisierte und überprüfbare Formen der Entlohnung »valuing care by >efficiency< standards«, wie Puig de la Bellacasa den

12 Vgl. Puig de la Bellacasa2015; Ploeg 2018b.

13 Vgl. Jurtschitsch 2010; Vogt 2000. 
Umstand umschreibt. ${ }^{14}$ Der Verwertung von care-Arbeit sind Grenzen gesetzt, insofern Sorgearbeit kaum Renditen bringen kann. Gleichzeitig baut die Gesellschaft darauf auf, dass care-Arbeiten dauerhaft kostengünstig zur Verfügung stehen. Eine sorgsame Landwirtschaft muss, genau wie andere Pflege- und Sorgearbeiten, Wege finden, um in einer marktorientierten Gesellschaft $\mathrm{zu}$ überleben. Die bisher üblichen Lösungswege - die Ansprüche zu verringern oder den Weg der Selbstausbeutung zu wählen - werden von den Bewirtschafter*innen bewusst abgelehnt, jedoch sind auch sie immer wieder dazu gezwungen, diese anzuwenden. Stattdessen wollen sie die ihnen zur Verfügung stehenden Ressourcen nutzen, um sich Freiräume zu schaffen, für einen lebenswerten Arbeitsalltag in der Landwirtschaft. Hier sind politische Veränderungen gefragt. Die Last und das Risiko, ein globales Lebensmittelsystem umzustrukturieren, kann nicht allein die Aufgabe bescheiden wirtschaftender Öko-Landwirt*innen sein. So liegt etwa die (nicht entgoltene) Bildungsarbeit Großteils bei ihnen, Bürokratie und Konsument*innen über den Wert einer sorgsamen Landwirtschaft zu unterrichten.

Wie Heistinger darstellt, gibt es aber auch Ressourcen, welche die Familien in vielerlei Hinsicht resilient machen, wie eine familiär erworbene Offenheit für (Betriebs-)Veränderungen, vielfältige, (auch informelle) Bildungswege und Berufserfahrungen, auch abseits der Landwirtschaft. Die Kombination unterschiedlicher Erfahrungen kann helfen, die Probleme der Landwirtschaft aus anderen Perspektiven zu betrachten, was in der Folge die Entwicklung neuer Lösungsstrategien begünstigt. Die neuen Landwirt*innen denken interdisziplinär. Sie können in einer komplexen Welt die Puzzlestücke finden, die sich zu einer erfolgreichen Betriebsstrategie zusammensetzen lassen. Die untersuchten Öko-Betriebe setzen dabei individuell gefundene Handlungspraxen um, die nicht unbedingt generalisierbar sind. Denn Innovationen sind regional angepasst und mit bestimmten Persönlichkeiten und deren familiären Ressourcen verknüpft. 
Heistinger arbeitet in ihrem Kapitel heraus, dass es kein spezifisches, abgrenzbares lokales Wissen gibt, das an einem Ort gefunden und nur dort tradiert wird. Regionale Einbettung ist nicht starr, sondern ein lebendiger Zusammenhang, der durch den beschleunigten Weltmarkt noch viel veränderlicher geworden ist. Da die Herausforderungen durch Markt und Klima ebenso wandelbar sind wie die zur Verfügung stehenden Technologien und die Ansprüche der Konsument*innen, muss stets neues Wissen generiert werden. "Die Innovationen passieren auf den Betrieben und nicht in den Schulen « stellt Herr Bergmann im Interview dazu fest (Interview am 2.3.2018). Die Betriebsleiter*innen sind regional und überregional gut vernetzt, bedienen sich der neuesten Kommunikationstechnologien und experimentieren mit neuen Techniken. Dabei haben sie großes Interesse daran, ihr Wissen unter Kund"innen und Kolleg*innen zu verbreiten und sich untereinander auszutauschen. Der freie Austausch von Wissen und Erfahrungen erzeugt auf diese Weise Vorteile für kleinstrukturierte, regionalspezifisch wirtschaftende Familienbetriebe.

In allen Fallbeispielen erhielten die Höfe neue Impulse von außen, als Partner*innen hinzukamen, bzw. die Familie einen Betrieb neu übernahm. Die Untersuchung der Familiengeschichten von Betriebsleiter*innen lässt die enge Verknüpfung von ökologischer Landwirtschaft mit persönlichem Erfahrungswissen und gemeinwohlorientierten Handlungsmustern sichtbar werden. Ihnen gemeinsam ist, dass sie ihre eigenen theoretischen Überlegungen und Überzeugungen sowie Inspirationen aus ihrem sozialen Umfeld und all jene Kompetenzen, die sie in ihrem bisherigen (Berufs-)leben erworben haben in ihrer landwirtschaftlichen Praxis neu zusammenführen. Sie schöpfen aus ihrer eigenen Geschichte und knüpfen Beziehungen zu Boden, Pflanzen und Tieren, sowie zu jenen Menschen, die sie mit ihren Produkten versorgen. Nicht selten müssen auch Ablehnung im direkten sozialen Umfeld - in der unmittelbaren Nachbarschaft oder innerhalb der eigenen Berufsgruppe - und behördliche Inflexibilität ertragen werden. Um diesen Widerständen gut begegnen zu können spielen die Bestärkung und Anerkennung durch Familienmitglieder eine wichtige Rolle, aber auch durch jene Menschen, die sie mit Lebensmitteln 
versorgen. Auch die Aufmerksamkeit für ihre neuen ökologischen und sozialen Praktiken, etwa von Expert*innen oder Vertreter*innen der Landwirtschaftskammern, stärkt ihre Resilienz.

Weitere Forschungen $\mathrm{zu}$ den Praktiker*innen einer sorgsamen Landwirtschaft sind notwendig, um ihre Rolle in der Gesellschaft genauer zu beleuchten, sowie ihre speziellen Bedürfnisse zu ergründen. Neue, kreative Ansätze der Analyse sind hier gefragt, die es erlauben, vielschichtige Dimensionen zu erkennen, wie sie in einer alternativen Lebensmittelproduktion und Distribution gegeben sind. In ihrem feministisch-kritischen Manifest Gens stellen Laura Bear u.a. fest: „Capitalism is at its core a diverse, intimate network of human and nonhuman relations ${ }^{15}$ und rufen dazu auf, die vielfältigen Beziehungsnetzwerke, die hinter dem stehen, was oft unhinterfragt unter dem Begriff des Kapitalismus zusammengefasst wird, zu erforschen. Mit einer System-Aufstellung zur Landwirtschaft begeht auch Heistinger neue kreative Wege in der Analyse. Zusammen mit der Künstlerin Susanne Schuda nähert sie sich der Frage »Wie können die Ressourcen des Agrarsystems in einander nährende Beziehungen kommen? « ${ }^{16}$ und zeigt dabei die Komplexität der Beziehungsnetzwerke zwischen menschlichen und nicht-menschlichen Akteur*innen in einem globalen Lebensmittelsystem auf.

Mit unterschiedlichen disziplinären Herangehensweisen beleuchten die Texte in diesem Band die verborgenen Annahmen über Sorgetragen in der Landwirtschaft und erkunden die ökologischen, sozialen, ökonomischen, politischen und affektiven Praxen einer sorgsamen Landwirtschaft als Beziehungen von Multi-Spezies Gemeinschaften, die nicht alleine Menschen zum Vorteil gereichen. Dabei werden die vielschichtigen lokalisierten Beziehungsgeflechte zwischen Menschen, Tieren, Pflanzen und Böden als mehr-als-menschliche Kollektive sichtbar. Damit soll der Band auch einen Beitrag zum Verständnis der Komplexität von Praktiken der Sorge leisten, im Sinne Puig de 
124 Elisabeth Kosnik, Andrea Heistinger, Gabriele Sorgo

la Bellacasas »als eine materielle Praxis des Verbindens und der politischen Intervention, die die unbeachteten und vernachlässigten Dinge mit einbezieht ${ }^{17}{ }^{17}$ als sozio-ökonomische Praktiken, politischgesellschaftliche Teilhabe und Umweltgerechtigkeit.

17 Gesing u.a. 2018, S. 36. 